This article may be downloaded for personal use only. Any other use requires prior permission of the author and AIP Publishing.

This article appeared in Surface Science Spectra 27, 024001 (2020) and may be found at https://doi.org/10.1116/6.0000270. 


\section{Bismuth acetate by XPS}

Jhonatan Rodriguez-Pereira ${ }^{1,2}$, Sergio A. Rincón-Ortiz ${ }^{1}$ and Rogelio Ospina ${ }^{1, a)}$

${ }^{1}$ Centro de Investigación Científica y Tecnológica en Materiales y Nanociencias (CMN), Universidad Industrial de Santander, Piedecuesta, Santander, P.C. 681011, Colombia.

${ }^{2}$ Center of Materials and Nanotechnologies, Faculty of Chemical Technology, University of Pardubice, Nam. Cs. Legii 565, 53002 Pardubice, Czech Republic.

(Received day Month year; accepted day Month year; published day Month year)

Bismuth (III) acetate was analyzed using x-ray photoelectron spectroscopy (XPS). The specimen is a powder purchased from Sigma Aldrich. Sample was fixed to a stainless-steel sample holder with copper $3 \mathrm{M}^{\mathrm{TM}}$ double-sided adhesive tape. Survey spectra, Bi 4f, O 1s, C 1s, Bi 4d, Bi 5d, $\mathrm{Bi}$ 6s core levels and valence band spectra were acquired. Results showed how the elements in the structure of bismuth acetate are related.

Keywords: Bismuth acetate; XPS; precursor; acetic acid bismuth
Accession\#: 01648

Technique: XPS

Host Material: Bismuth acetate

Instrument: SPECS

PHOIBOS 150

Major Elements in Spectra: Bi, O, C

Minor Elements in Spectra: None

Published Spectra: 6

Spectra in Electronic Record: 6

Spectral Category: comparison

\section{INTRODUCTION}

Bismuth (III) acetate is an inorganic compound consisting of positive bismuth ions (3+ charge) and negative acetate ions (1charge) (Fig. 1). It is commonly employed as a precursor for the synthesis of several materials with different applications such as, bismuth sulfide for solar cells (Refs. 1 and 2), bismuth titanate nanorods as photocatalysts (Refs. 3 - 5), sensitization of $\mathrm{TiO}_{2}$ nanotubes on photoelectrochemical reactions (Ref. 6) and it is also used in the synthesis of triarylbismuth compounds (Ref. 7).

\section{C}

Fig. 1. Structure of bismuth acetate

XPS survey spectrum shows that the sample does not present intrusive elements, since the presence of only carbon, oxygen and bismuth is evidenced.

$\mathrm{Bi}$ 4f high resolution spectrum was decomposed in four contributions corresponding to two different chemical environments. The peaks centered at 159.3 and $164.6 \mathrm{eV}$ were assigned to bismuth bonding with oxygen, O-Bi-O, and signals at 160.9 and $166.2 \mathrm{eV}$ were associated with bismuth bonding with oxygen and the last one with carbon, Bi-O-C. O 1s spectrum displayed five different species. First, at $530.3 \mathrm{eV}$ corresponds to oxygen bonding with bismuth, O-Bi-O. Second, carbon double and single bonded with oxygen, $\mathbf{O}=\mathrm{C}-\mathbf{O}$ at $531.1 \mathrm{eV}$. Third, at
$531.8 \mathrm{eV}$ was assigned to $(\mathrm{C}=\mathbf{O})-\mathrm{O}-\mathrm{Bi}$ species. Fourth, oxygen single bounded with carbon, C-O at $532.6 \mathrm{eV}$. And finally, 533.4 $\mathrm{eV}$ has an overlap between $(\mathrm{C}=\mathrm{O})-\mathbf{O}-\mathrm{Bi}$ and adsorbed $\mathbf{O H}$. High resolution spectrum of $\mathrm{C} 1 \mathrm{~s}$ shows four chemical species. C$(\mathrm{C}, \mathrm{H})$ at $284.8 \mathrm{eV}$, carbon carbon bonding or adventitious carbon used as a reference (Ref. 8). Carbon single bounded with oxygen, C-O at $286.3 \mathrm{eV}$. Carbon double and single bonded with oxygen, $\mathrm{O}=\mathbf{C}-\mathrm{O}$ at $288.4 \mathrm{eV}$, and carbon double bonded with oxygen related with bismuth, $(\mathbf{C}=\mathrm{O})-\mathrm{O}-\mathrm{Bi}$ at $290 \mathrm{eV}$. Bi 4d and $\mathrm{Bi} 5 \mathrm{~d}$ signals were also fitted to confirm the surface chemical state found in $\mathrm{Bi} 4 \mathrm{f}$. The results of this work evidenced the bonds between bismuth, oxygen and carbon in the structure of bismuth acetate.

\section{SPECIMEN DESCRIPTION (ACCESSION \# 01648)}

Host Material: Bismuth acetate

CAS Registry \#: 22306-37-2

Host Material Characteristics: homogeneous; solid; polycrystalline; unknown conductivity; inorganic compound; Powder

Chemical Name: Bismuth triacetate

Source: Sigma Aldrich

Host Composition: Bismuth (III) acetate (99.99\%)

Form: Powder

Structure: $\mathrm{Bi}\left(\mathrm{CH}_{3} \mathrm{COO}\right)_{3}$

History \& Significance: Bismuth (III) acetate powder was ground and fixed to a sample holder with copper $3 \mathrm{M}^{\mathrm{TM}}$ doublesided adhesive tape. The sample was exposed to the environment for about 2 minutes, time that was spent to prepare the sample and then introduce it to the platform.

As Received Condition: As powder

Analyzed Region: same as host material

Ex Situ Preparation/Mounting: As received. 
In Situ Preparation: None

Charge Control: Electron flood gun (SPECS FG-500) operated at $70 \mu \mathrm{A}$ and $4 \mathrm{eV}$

Temp. During Analysis: $300 \mathrm{~K}$

Pressure During Analysis: $<1 \times 10^{-7} \mathrm{~Pa}$

Pre-analysis Beam Exposure: Not applicable $s$

\section{INSTRUMENT DESCRIPTION}

Manufacturer and Model: SPECS PHOIBOS 150 - 2D-DLD - SPECS Surface Nano Analysis GmbH

Analyzer Type: spherical sector

Detector: other

Number of Detector Elements: 25

\section{INSTRUMENT PARAMETERS COMMON TO ALL SPECTRA}

\section{-Spectrometer}

Analyzer Mode: constant pass energy

Throughput $\left(T=E^{N}\right): N=0$

Excitation Source Window: Mylar window, allows high Xray transmission: $88 \%$ for $\mathrm{Al} \mathrm{K} \alpha$.

Excitation Source: Al Ka monochromatic

Source Energy: $1486.6 \mathrm{eV}$

Source Strength: $200 \mathrm{~W}$

Source Beam Size: $2000 \mu \mathrm{m}$ x $2000 \mu \mathrm{m}$

Signal Mode: multichannel direct

-Geometry

Incident Angle: $55^{\circ}$

Source-to-Analyzer Angle: $55^{\circ}$

Emission Angle: $0^{\circ}$

Specimen Azimuthal Angle: Not applicable

Acceptance Angle from Analyzer Axis: $16^{\circ}$

Analyzer Angular Acceptance Width: $16^{\circ}$ x $16^{\circ}$

-lon Gun

Manufacturer and Model: SPECS IQE 12/38

Energy: $5000 \mathrm{eV}$

Current: $70 \mathrm{~mA}$

Current Measurement Method: biased stage

Sputtering Species: $\mathrm{Ar}^{+}$

Spot Size (unrastered): $3000 \mu \mathrm{m}$ x $3000 \mu \mathrm{m}$

Raster Size: Not applicable $\mu \mathrm{m}$ x $\mu \mathrm{m}$

Incident Angle: $54^{\circ}$

Polar Angle: $55^{\circ}$

Azimuthal Angle: $45^{\circ}$

Comment: The specimen was analyzed as loaded. The ion gun was used only for cleaning the Ag reference foil.
Energy Scale Correction: Binding energy of the adventitious carbon, C-(C,H) at $284.8 \mathrm{eV}$ (Ref. 8) was used as reference to adjust the binding energy scale of the spectra.

Recommended Energy Scale Shift: $2.54 \mathrm{eV}$

Peak Shape and Background Method: Peak position and width were determined from fitting the spectra using a mixed Gaussian- Lorentzian, GL (30) function after subtraction of a Shirley background using the CasaXPS Software.

Quantitation Method: Peak areas were obtained from fitting the spectra and relative sensitivity factors from the atomic photoionization cross section of each core level provided by SPECS Prodigy library.

\section{ACKNOWLEDGMENTS}

Authors thank to Laboratorio Central en Ciencia de Superficies (SurfLab-UIS) from Universidad Industrial de Santander for providing their facilities to perform the XPS measurements and to project 2321 of Vicerrectoría de Investigación y Extensión (VIE-UIS) from Universidad Industrial de Santander.

\section{REFERENCES}

1. J.H. Kim, H. Park, C.H. Hsu and J. Xu, J. Phys. Chem. C., 114, 9634 (2010).

2. L. Martinez, A. Stavrinadis, S. Higuchi, S.L. Diedenhofen, M. Bernechea, K. Tajima and G Konstantatos, Phys.Chem.Chem.Phys., 15, 5482 (2013).

3. L.Z. Pei, H.D. Liu, N. Lin and H.Y. Yu, J. Alloys Compd., 622, 254 (2015).

4. S. Murugesan and V. Subramanian, Chem. Commun., 5109 (2009).

5. J. Hou, S. Jiao, H. Zhu and R.V. Kumar, CrystEngComm, 13, 4735 (2011)

6. L.J. Hoyos, D.F. Rivera, A.F. Gualdrón-Reyes, R. Ospina, J. Rodriguez-Pereira, J.L. Ropero-Vega and M.E. Niño-Gomez, Appl. Surf. Sci., 423, 917 (2017).

7. V. Stavila, J.H. Thurston, D. Prieto-Centurión and K.H. Whitmire, Organometallics, 26, 6864 (2007).

8. P. G. Rouxhet and M. J. Genet, Surf. Interface Anal. 43, 1453 (2011). 


\begin{tabular}{|c|c|c|c|c|c|c|c|}
\hline \multicolumn{8}{|c|}{ SPECTRAL FEATURES TABLE } \\
\hline $\begin{array}{c}\text { Spectrum } \\
\text { ID \# }\end{array}$ & $\begin{array}{l}\text { Element/ } \\
\text { Transition }\end{array}$ & $\begin{array}{c}\text { Peak } \\
\text { Energy } \\
(\mathrm{eV})\end{array}$ & $\begin{array}{c}\text { Peak } \\
\text { Width } \\
\text { FWHM } \\
(\mathrm{eV}) \\
\end{array}$ & $\begin{array}{l}\text { Peak Area } \\
(\mathrm{eV} \times \mathrm{cts} / \mathrm{s})\end{array}$ & $\begin{array}{l}\text { Sensitivity } \\
\text { Factor }\end{array}$ & $\begin{array}{l}\text { Concentration } \\
\text { (at. \%) }\end{array}$ & Peak Assignment \\
\hline 01648-02 & $\mathrm{Bi} 4 \mathrm{f}$ & $\ldots$ & & $2.98 \times 10^{4}$ & 25.66 & 15.28 & \\
\hline 01648-02 & $\mathrm{Bi} 4 \mathrm{f}_{7 / 2}$ & 159.3 & 1.52 & $\ldots$ & $\ldots$ & $\ldots$ & O-Bi-O \\
\hline 01648-02 & Bi $4 f_{5 / 2}$ & 164.6 & 1.52 & $\ldots$ & $\cdots$ & $\ldots$ & O-Bi-O \\
\hline 01648-02 & $\mathrm{Bi} 4 \mathrm{f}_{7 / 2}$ & 160.9 & 1.90 & $\ldots$ & $\ldots$ & $\ldots$ & $\mathrm{Bi}-\mathrm{O}-\mathrm{C}$ \\
\hline 01648-02 & Bi $4 f_{5 / 2}$ & 166.2 & 1.90 & $\ldots$ & $\ldots$ & $\ldots$ & Bi-O-C \\
\hline 01648-03 & 0 1s & $\ldots$ & $\ldots$ & $7.49 \times 10^{3}$ & 2.77 & 40.15 & $\ldots$ \\
\hline $01648-03$ & 015 & 530.3 & 2.00 & $\ldots$ & $\ldots$ & $\ldots$ & O-Bi-O \\
\hline 01648-03 & 0 1s & 531.1 & 2.00 & $\ldots$ & $\ldots$ & $\ldots$ & $\mathrm{O}=\mathrm{C}-\mathrm{O}$ \\
\hline 01648-03 & $01 \mathrm{~s}$ & 531.8 & 2.00 & $\ldots$ & $\ldots$ & $\ldots$ & $(\mathrm{C}=\mathrm{O})-\mathrm{O}-\mathrm{Bi}$ \\
\hline $01648-03$ & $01 s$ & 532.6 & 2.00 & $\ldots$ & $\ldots$ & $\ldots$ & C-O \\
\hline 01648-03 & 0 1s & 533.4 & 2.00 & $\ldots$ & $\ldots$ & $\ldots$ & $(\mathrm{C}=\mathrm{O})-\mathbf{O}-\mathrm{Bi}, \mathrm{OH}$ \\
\hline 01648-04 & C 1s & $\ldots$ & $\ldots$ & $3.30 \times 10^{3}$ & 1.00 & 44.57 & $\ldots$ \\
\hline 01648-04 & C 1s & 284.8 & 1.74 & $\ldots$ & $\ldots$ & $\ldots$ & C- $(\mathrm{C}, \mathrm{H})$ \\
\hline 01648-04 & C 1s & 286.3 & 1.74 & $\ldots$ & $\ldots$ & $\ldots$ & C-O \\
\hline 01648-04 & C 1s & 288.4 & 1.74 & $\ldots$ & $\ldots$ & $\ldots$ & $\mathrm{O}=\mathbf{c}-\mathrm{O}$ \\
\hline 01648-04 & C 1s & 290.0 & 1.74 & $\ldots$ & $\ldots$ & $\ldots$ & $(\mathbf{C}=\mathrm{O})-\mathrm{O}-\mathrm{Bi}$ \\
\hline 01648-05 & $\mathrm{Bi} 4 \mathrm{~d}$ & $\ldots$ & $\ldots$ & $1.90 \times 10^{4}$ & $\ldots$ & $\ldots$ & $\ldots$ \\
\hline 01648-05 & $\mathrm{Bi} 4 d_{5 / 2}$ & 442.1 & 4.00 & $\ldots$ & $\ldots$ & $\ldots$ & O-Bi-O \\
\hline 01648-05 & $\mathrm{Bi} 4 d_{3 / 2}$ & 465.9 & 4.00 & $\ldots$ & $\ldots$ & $\ldots$ & O-Bi-O \\
\hline 01648-05 & $\mathrm{Bi} 4 d_{5 / 2}$ & 444.4 & 3.50 & $\ldots$ & $\ldots$ & $\ldots$ & $\mathrm{Bi}-\mathrm{O}-\mathrm{C}$ \\
\hline 01648-05 & $\mathrm{Bi} 4 d_{3 / 2}$ & 468.2 & 3.50 & $\ldots$ & $\ldots$ & $\ldots$ & $\mathrm{Bi}-\mathrm{O}-\mathrm{C}$ \\
\hline 01648-06 & Bi $5 d$ & $\ldots$ & $\ldots$ & $4.14 \times 10^{3}$ & $\ldots$ & $\ldots$ & $\ldots$ \\
\hline 01648-06 & Bi $5 d_{5 / 2}$ & 26.1 & 1.58 & $\ldots$ & $\ldots$ & $\ldots$ & O-Bi-O \\
\hline 01648-06 & $\mathrm{Bi} 5 d_{3 / 2}$ & 29.1 & 1.58 & $\ldots$ & $\ldots$ & $\ldots$ & O-Bi-O \\
\hline 01648-06 & Bi $5 d_{5 / 2}$ & 27.9 & 2.00 & $\ldots$ & $\ldots$ & $\ldots$ & $\mathrm{Bi}-\mathrm{O}-\mathrm{C}$ \\
\hline 01648-06 & $\mathrm{Bi} 5 d_{3 / 2}$ & 30.9 & 2.00 & $\ldots$ & $\ldots$ & $\ldots$ & $\mathrm{Bi}-\mathrm{O}-\mathrm{C}$ \\
\hline 01648-06 & Bi $6 s$ & 11.7 & 2.99 & $0.74 \times 10^{2}$ & $\ldots$ & $\ldots$ & $\ldots$ \\
\hline $01648-06^{a}$ & VBM & 1.73 & $\ldots$ & $\ldots$ & $\ldots$ & $\ldots$ & $\ldots$ \\
\hline
\end{tabular}

a Valence band maximum (VBM)

\begin{tabular}{|c|c|c|c|c|c|c|c|}
\hline \multicolumn{8}{|c|}{ ANALYZER CALIBRATION TABLE } \\
\hline $\begin{array}{c}\text { Spectrum ID } \\
\#\end{array}$ & $\begin{array}{l}\text { Element/ } \\
\text { Transition }\end{array}$ & $\begin{array}{c}\text { Peak Energy } \\
(\mathrm{eV})\end{array}$ & $\begin{array}{l}\text { Peak Width } \\
\text { FWHM (eV) }\end{array}$ & $\begin{array}{c}\text { Peak Area } \\
\text { (eV x cts/s) }\end{array}$ & $\begin{array}{l}\text { Sensitivity } \\
\text { Factor }\end{array}$ & $\begin{array}{c}\text { Concentration } \\
\text { (at. \%) }\end{array}$ & $\begin{array}{c}\text { Peak } \\
\text { Assignment }\end{array}$ \\
\hline$\ldots$ & $\mathrm{Ag} \mathrm{3d} 5 / 2$ & 368.3 & 0.50 & $0.15 \times 10^{6}$ & & & $\ldots$ \\
\hline
\end{tabular}


GUIDE TO FIGURES

\begin{tabular}{|c|c|c|c|c|c|}
\hline $\begin{array}{l}\text { Spectrum } \\
\text { (Accession) \# }\end{array}$ & Spectral Region & Voltage Shift* & Multiplier & Baseline & Comment \# \\
\hline $01648-01$ & Survey & 0 & 1 & 0 & 1 \\
\hline 01648-02 & $\mathrm{Bi} 4 \mathrm{f}$ & -2.54 & 1 & 0 & 1 \\
\hline 01648-03 & $01 \mathrm{~s}$ & -2.54 & 1 & 0 & 1 \\
\hline 01648-04 & C 1s & -2.54 & 1 & 0 & 1 \\
\hline 01648-05 & $\mathrm{Bi} 4 \mathrm{~d}$ & -2.54 & 1 & 0 & 1 \\
\hline 01648-06 & Bi $5 d$, Bi $6 s$, VB & -2.54 & 1 & 0 & 1 \\
\hline
\end{tabular}

*Voltage shift of the archived (as-measured) spectrum relative to the printed figure. The figure reflects the recommended energy scale correction due to a calibration correction, sample charging, flood gun, or other phenomenon.

1, Bismuth (III) acetate powder 


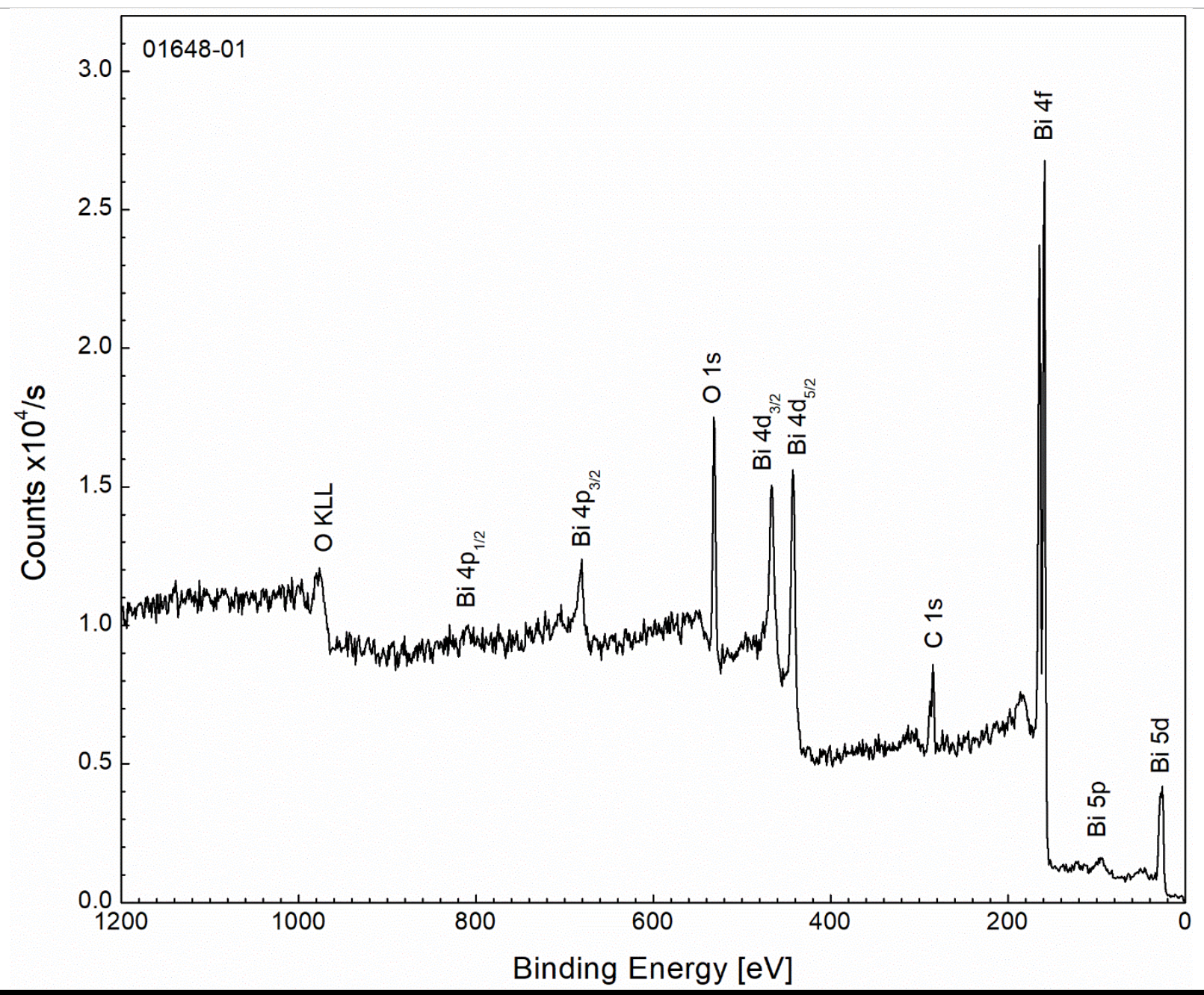

Publish in Surface Science Spectra: Yes $\otimes$ No $\square$

Accession \# 01648-01

Host Material

Technique

Spectral Region

Instrument

Excitation Source

Source Energy

Source Strength

Source Size

Analyzer Type

Incident Angle

Emission Angle

Analyzer Pass Energy

Analyzer Resolution

Total Signal Accumulation Time

Total Elapsed Time

Number of Scans
Bismuth acetate

XPS

survey

SPECS PHOIBOS 150

$\mathrm{Al}$ Ka monochromatic

$$
\begin{gathered}
1486.6 \mathrm{eV} \\
200 \mathrm{~W} \\
2 \mathrm{~mm} \times 2 \mathrm{~mm}
\end{gathered}
$$

spherical sector analyzer

$55^{\circ}$
$0^{\circ}$
$100 \mathrm{eV}$
$1.7 \mathrm{eV}$
$122 \mathrm{~s}$
$260 \mathrm{~s}$
1
$5.28 \mathrm{eV}$




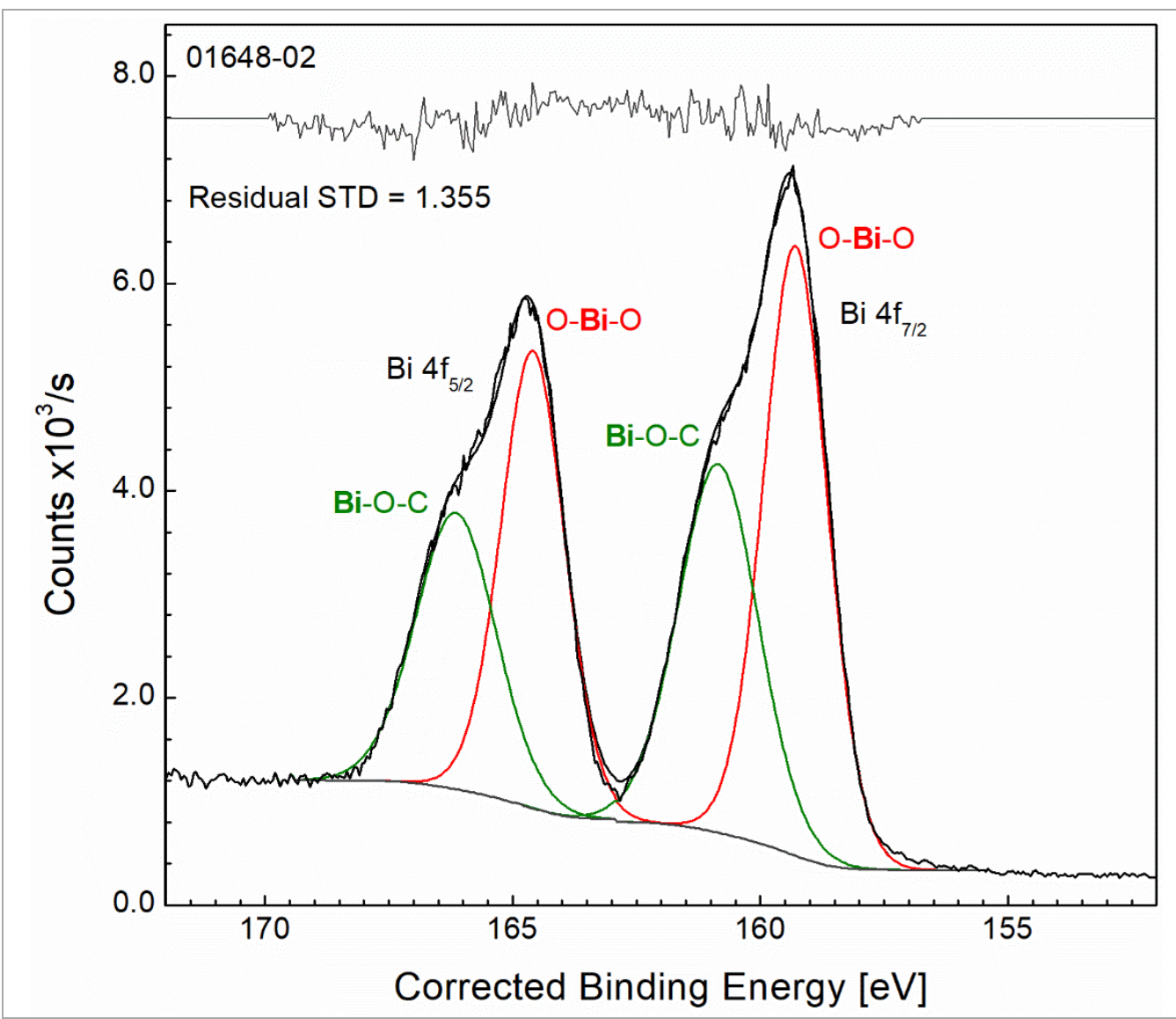

Publish in SSS: Yes $\otimes$ No $\square$

- Accession \#: 01648-02

- Host Material: Bismuth acetate

- Technique: XPS

- Spectral Region: Bi $4 f$ Instrument: SPECS PHOIBOS 150

Excitation Source: Al Ka monochromatic

Source Energy: $1486.6 \mathrm{eV}$

Source Strength: $200 \mathrm{~W}$

Source Size: $2 \mathrm{~mm} \times 2 \mathrm{~mm}$

Analyzer Type: spherical sector

Incident Angle: $55^{\circ}$

Emission Angle: $0^{\circ}$

Analyzer Pass Energy $30 \mathrm{eV}$

Analyzer Resolution: $0.5 \mathrm{eV}$

Total Signal Accumulation Time: $601 \mathrm{~s}$

Total Elapsed Time: $954 \mathrm{~s}$

Number of Scans: 8

Effective Detector Width: $2.64 \mathrm{eV}$

Publish in SSS: Yes $\otimes$ No $\square$

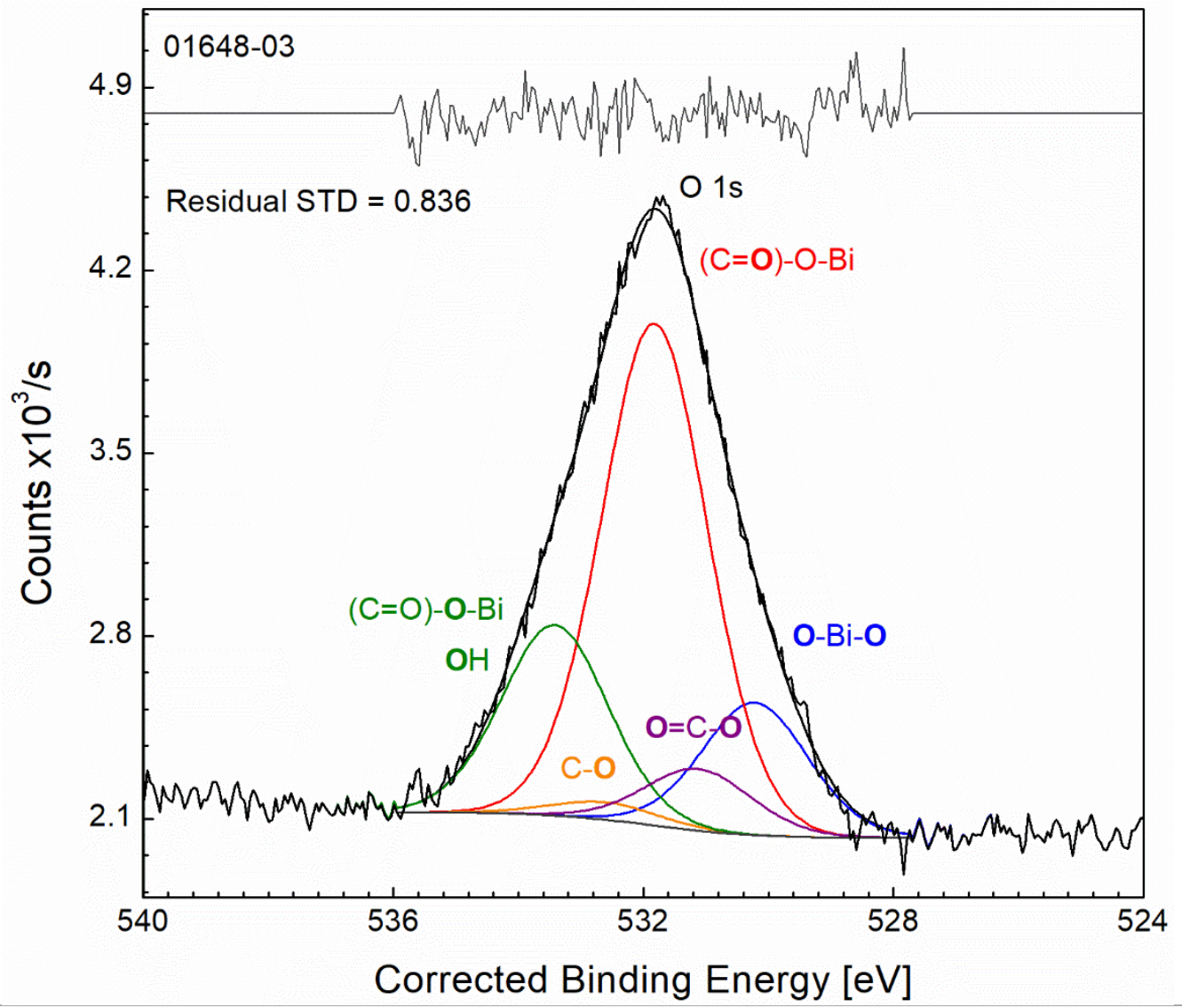

- Accession \#: 01648-03

- Host Material: Bismuth acetate

- Technique: XPS

- Spectral Region: O 1s Instrument: SPECS PHOIBOS 150

Excitation Source: Al Ka monochromatic Source Energy: $1486.6 \mathrm{eV}$ Source Strength: $200 \mathrm{~W}$ Source Size: $2 \mathrm{~mm} \times 2 \mathrm{~mm}$ Analyzer Type: spherical sector Incident Angle: $55^{\circ}$ Emission Angle: 0 Analyzer Pass Energy $30 \mathrm{eV}$ Analyzer Resolution: $0.5 \mathrm{eV}$ Total Signal Accumulation Time: 421 $\mathrm{s}$ Total Elapsed Time: $668 \mathrm{~s}$ Number of Scans: 8 Effective Detector Width: $2.64 \mathrm{eV}$ 


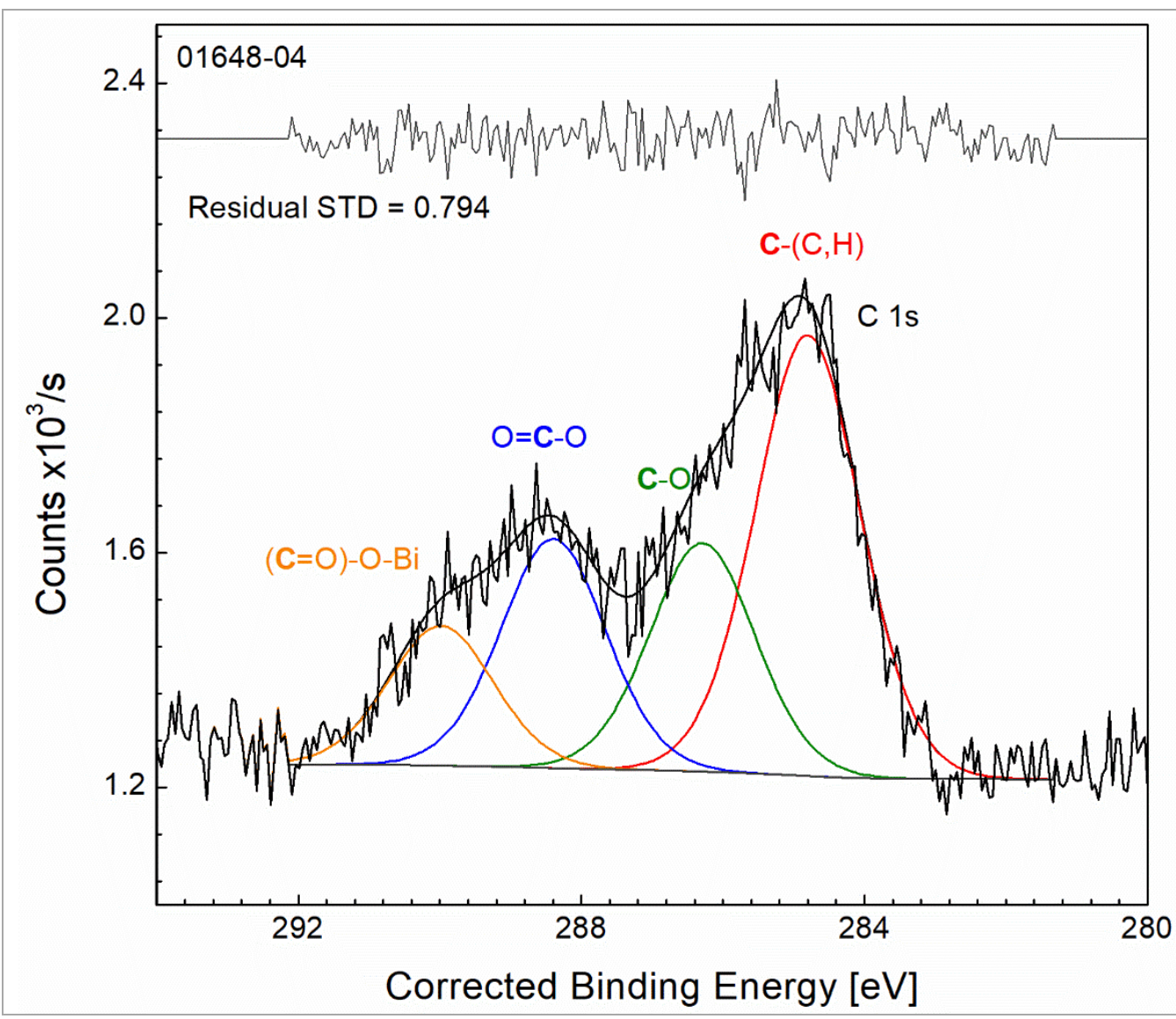

Publish in SSS: Yes $\otimes$ No $\square$

- Accession \#: 01648-04

- Host Material: Bismuth acetate

- Technique: XPS

- Spectral Region: C 1s Instrument: SPECS PHOIBOS 150

Excitation Source: $\mathrm{Al} \mathrm{Ka}$ monochromatic

Source Energy: $1486.6 \mathrm{eV}$

Source Strength: $200 \mathrm{~W}$

Source Size: $2 \mathrm{~mm} \times 2 \mathrm{~mm}$

Analyzer Type: spherical sector Incident Angle: $55^{\circ}$

Emission Angle: $0^{\circ}$

Analyzer Pass Energy $30 \mathrm{eV}$ Analyzer Resolution: $0.5 \mathrm{eV}$

Total Signal Accumulation Time: 211 $\mathrm{s}$

Total Elapsed Time: 335 s

Number of Scans: 4

Effective Detector Width: $2.64 \mathrm{eV}$

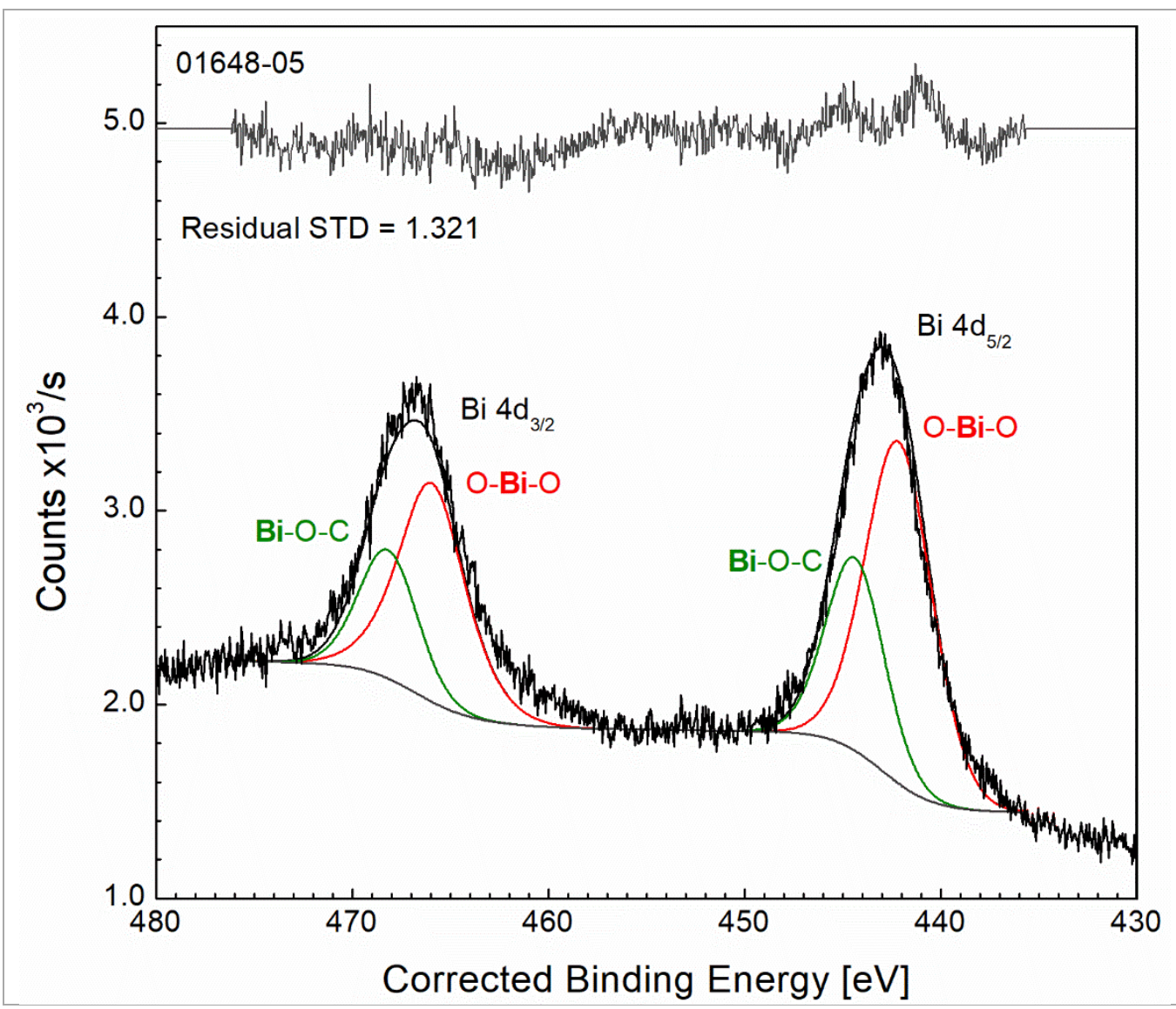

Publish in SSS: Yes $\triangle$ No $\square$

- Accession \#: 01648-05

- Host Material: Bismuth acetate

- Technique: XPS

- Spectral Region: Bi 4d Instrument: SPECS PHOIBOS 150

Excitation Source: Al Ka monochromatic

Source Energy: $1486.6 \mathrm{eV}$

Source Strength: $200 \mathrm{~W}$

Source Size: $2 \mathrm{~mm} \times 2 \mathrm{~mm}$

Analyzer Type: spherical sector Incident Angle: $55^{\circ}$

Emission Angle: $0{ }^{\circ}$

Analyzer Pass Energy $30 \mathrm{eV}$

Analyzer Resolution: $0.5 \mathrm{eV}$

Total Signal Accumulation Time: 1361

$\mathrm{s}$

Total Elapsed Time: $2160 \mathrm{~s}$

Number of Scans: 4

Effective Detector Width: $2.64 \mathrm{eV}$ 


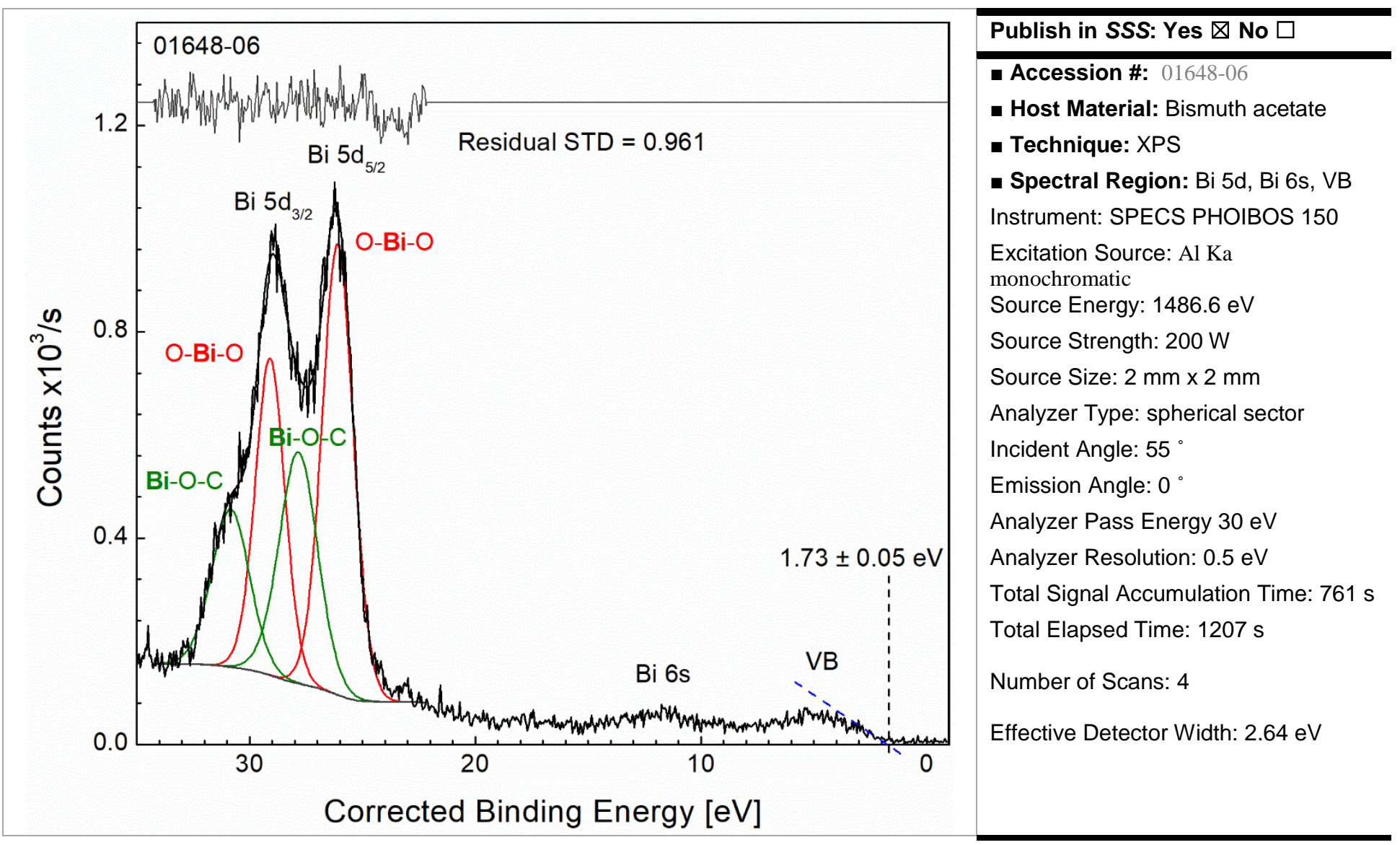

